\title{
Expression Profiles of NOS Isoforms in Dental Pulp and Odontoblasts in nNOS Knockout Mice
}

\author{
Yoichi Ishizuka $^{\text {1) }}$, Mizuki Yoshida ${ }^{2)}$, Kimiharu Ambe ${ }^{3)}$, Junzo Sasaki ${ }^{4)}$, \\ Naoki Sugihara ${ }^{1)}$ and Hiroki Watanabe ${ }^{3)}$
}

${ }^{1)}$ Department of Epidemiology and Public Health, Tokyo Dental College, 2-9-18 Kanda-Misakicho, Chiyoda-ku, Tokyo 101-0061, Japan

2) Gunma Oral Health Center for Special Needs Dentistry, 1-5-17 Otomo-machi, Maebashi, Gunma 371-0847, Japan

3) Division of Oral Histology, Department of Morphological Biology, Ohu University School of Dentistry, 31-1 Misumido, Tomita-machi, Koriyama, Fukushima 963-8611, Japan

${ }^{4)}$ Department of Nursing, Niimi University, 1263-2 Nishigata, Niimi, Okayama 718-8585, Japan

Received 10 January, 2019/Accepted for Publication 8 February, 2019 Published Online in J-STAGE 23 November, 2019

\begin{abstract}
Active oxygen and free radicals are involved in metabolism in cells and tissues. Immunohistological studies of related enzymes are few, and the morphological dynamics of these enzymes in dental pulp and odontoblasts remain to be elucidated. Nitric oxide synthase (NOS) has 3 isoforms: nNOS, iNOS, and eNOS. The aim of this study was to investigate the profiles of NOS isoforms in the absence of nNOS in dental pulp and odontoblasts. Five-week-old male C57BL/6 and nNOS knockout (KO) mice were sacrificed and expression of nNOS, iNOS, and eNOS determined immunohistochemically. Expression of nNOS was positive, whereas that of iNOS was negative and eNOS weakly positive in the dental pulp and odontoblasts of the control mice. In nNOS KO mice, expression of iNOS was positive in dental pulp and strongly positive in odontoblasts, whereas that of eNOS was stronger in fibroblasts, endothelial cells in the vicinity of blood vessels in the dental pulp, and odontoblasts. Expression of nNOS was negative in the nNOS KO mice. This suggests that iNOS and eNOS compensate for nNOS deficiency in vascular endothelial cells and fibroblasts in the dental pulp and odontoblasts.
\end{abstract}

Key words: Nitric oxide synthase - Knockout mice -

Dental pulp-Odontoblasts - Immunohistochemistry 


\section{Introduction}

Nitric oxide (NO), reactive oxygen, and antioxidative enzymes are induced together or inhibit each other, forming a complex network, and NO is closely involved in not only various diseases $^{2)}$, but also a wide range of physiological functions, including neurotransmission $^{17)}$, immune response ${ }^{11)}$, adjustment to the vascular system ${ }^{7)}$, apoptosis ${ }^{3)}$, and wound healing ${ }^{9}$.

Nitric oxide is synthesized by NO synthase (NOS) in the body, of which three distinct isoforms have been reported in mammalian tissues $^{6,8}$ : neuronal NOS (nNOS), which is constantly expressed in neurons; endothelial NOS (eNOS), which is expressed in vascular endothelial cells; and inductive NOS (iNOS) which is induced in inflammation reactions. These three isoforms compensate each other ${ }^{19,20)}$, and their clarification has enabled elucidation of specific individual NO production systems.

Reactive oxygen and free radicals are believed to be involved in metabolism and transport in the tissues of the oral cavity, particularly in endothelial cells and fibroblasts in the dental pulp, but the dynamics of the related enzymes remain to be clarified ${ }^{1)}$. Only eNOS and iNOS have been investigated in immunohistochemical studies of endothelial cells and fibroblasts in human dental pulp: the results showed that they were positive for $\operatorname{eNOS}^{4,5)}$, but negative for iNOS ${ }^{4)}$. While the presence of nNOS has been suggested based on investigation of NADPH- $\mathrm{d}^{14)}$, this remains to be demonstrated. Although iNOS and eNOS have been shown to compensate for nNOS deficiency in the gingiva of nNOS knockout mice ${ }^{13)}$, the relationship between cells expressing nNOS, iNOS, and eNOS and their synthases in dental pulp and odontoblasts remain to be clarified.

In the present study, it was hypothesized that iNOS and eNOS compensated for nNOS deficiency in dental pulp and odontoblasts. To investigate the dynamics of the other NO synthases in the absence of nNOS, expression of nNOS, iNOS, and eNOS was immunohisto- chemically investigated in vascular endothelial cells and fibroblasts in dental pulp and odontoblasts using nNOS knockout (KO) mice.

\section{Materials and Methods}

\section{Animal treatment}

All animals in these experiments were maintained and used in accordance with the Guidelines for the Care and Use of Laboratory Animals prepared by the Institute of Laboratory Animal Resources and the Guidelines for the Treatment of Experimental Animals at Tokyo Dental College (No.160204).

\section{Specimen preparation}

The control and experimental groups comprised 6 C57BL/ 6 mice and $6 \mathrm{nNOS} \mathrm{KO} \mathrm{mice}$ (Stock no.2633. Jackson Lab., Bar Harbor, USA $)^{12)}$, respectively. All mice were 5 -week-old males weighing approximately 20-25 g each. None of the mice in either the experimental or control group showed any evidence of inflammation, such as flare or swelling.

The animals were anesthetized with an overdose of thiopental (Somnopenthyl $64.8 \mathrm{mg} / \mathrm{ml}$ : Kyoritsu Seiyaku, Tokyo, Japan) diluted to $10 \%$ in saline and administered at $0.05 \mathrm{ml}$ per $10 \mathrm{~g}$ weight intraperitoneally. The maxilla and molar teeth (M1) were then excised and fixed by perfusion with $4 \%$ paraformaldehyde solution. They were then cut into $3.0-\mathrm{mm}^{3}$ pieces and fixed in $4 \%$ paraformaldehyde solution at room temperature for $2 \mathrm{hr}$. After washing, the samples were decalcified with $10 \%$ EDTA at $4^{\circ} \mathrm{C}$ for 2 weeks, dehydrated with alcohol by the conventional method, and then embedded in paraffin for preparation of thin sections.

\section{Immunohistochemistry}

Protein expression levels were investigated by immunohistochemistry. The paraffin sections were deparaffinized with xylene and ethanol and then reacted with glycine PBS for $30 \mathrm{~min}$. After that, they were reacted with $0.1 \% \mathrm{NaN}_{3}$ for 30 min and washed with PBS 


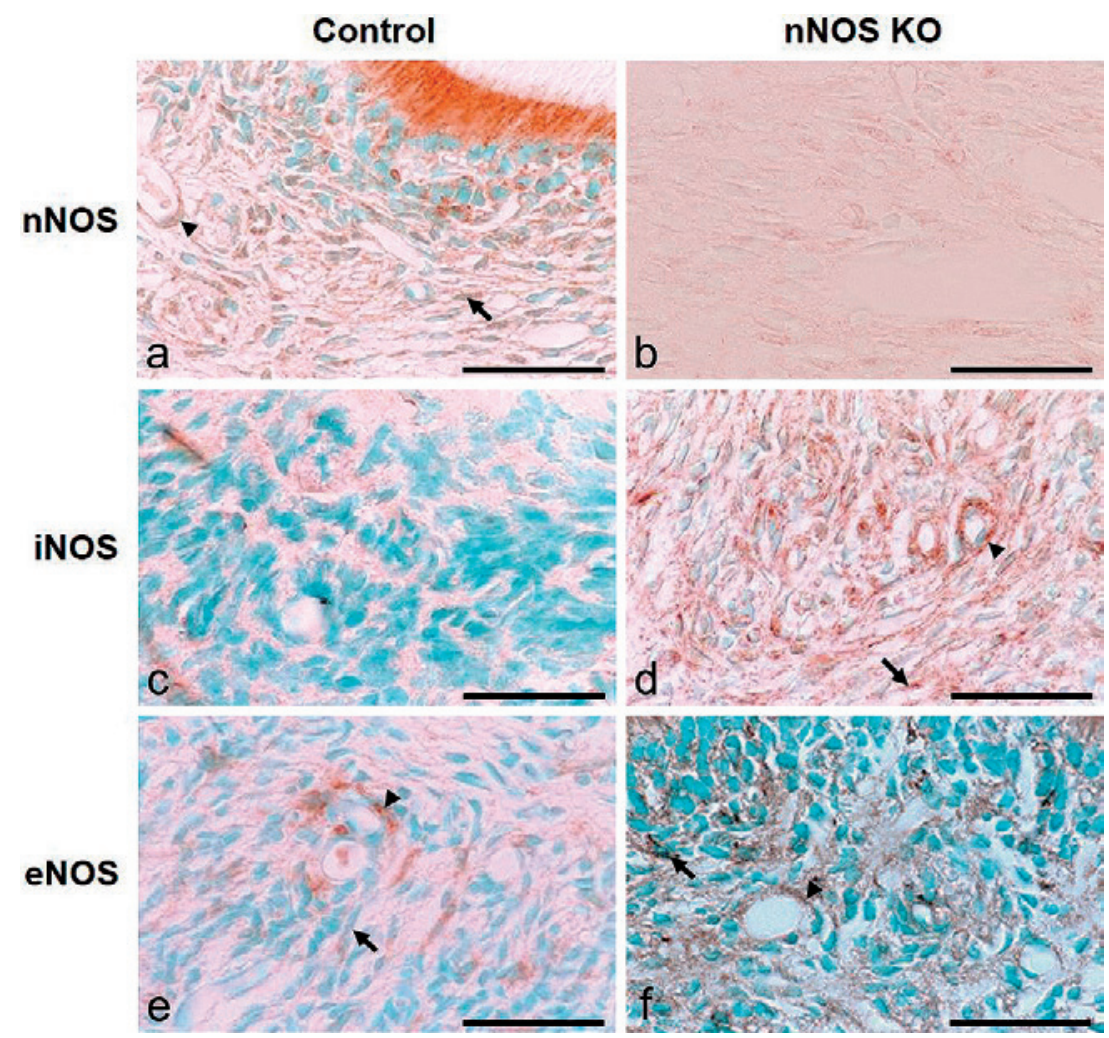

Fig. 1 NOS protein expression in central region of dental pulp

Positive reaction for nNOS was detected in endothelial cells (arrowhead) and fibroblasts (arrow) in vicinity of blood vessels in control group (a). Negative reaction for nNOS was observed in endothelial cells and fibroblasts in vicinity of blood vessels in nNOS KO group (b).

Negative iNOS expression was observed in control group (c). Positive reaction for iNOS was detected in endothelial cells (arrowhead) and fibroblasts (arrow) in vicinity of blood vessels in nNOS KO group (d).

As for eNOS, weakly positive reaction was noted in endothelial cells (arrowhead) and fibroblasts (arrow) in vicinity of blood vessels in control group. Strongly positive reaction was observed in endothelial cells (arrowhead) and fibroblasts (arrow) in vicinity of blood vessels in nNOS KO group.

Scale bar $=50 \mu \mathrm{m}$

Immunopositive staining is indicated by a dark brown color.

buffer. After washing, they were incubated in $1 \%$ non-fat dry milk for blocking for $1 \mathrm{hr}$ at room temperature. They were then washed with PBS buffer again and reacted with the primary antibody. For nNOS staining, rabbit anti-nNOS polyclonal IgG was used ${ }^{15,16)}$; for iNOS, rabbit anti-iNOS polyclonal IgG was used $^{18,21)}$; for eNOS, mouse anti-eNOS monoclonal IgG was used ${ }^{10,12)}$.

Sections were inspected using a Universal
Microscope: Axiophot 2 and AxioCam (ZEISS).

\section{Results}

A positive reaction revealing expression of nNOS was detected in endothelial cells and fibroblasts in the vicinity of blood vessels in the central region of the dental pulp in the 


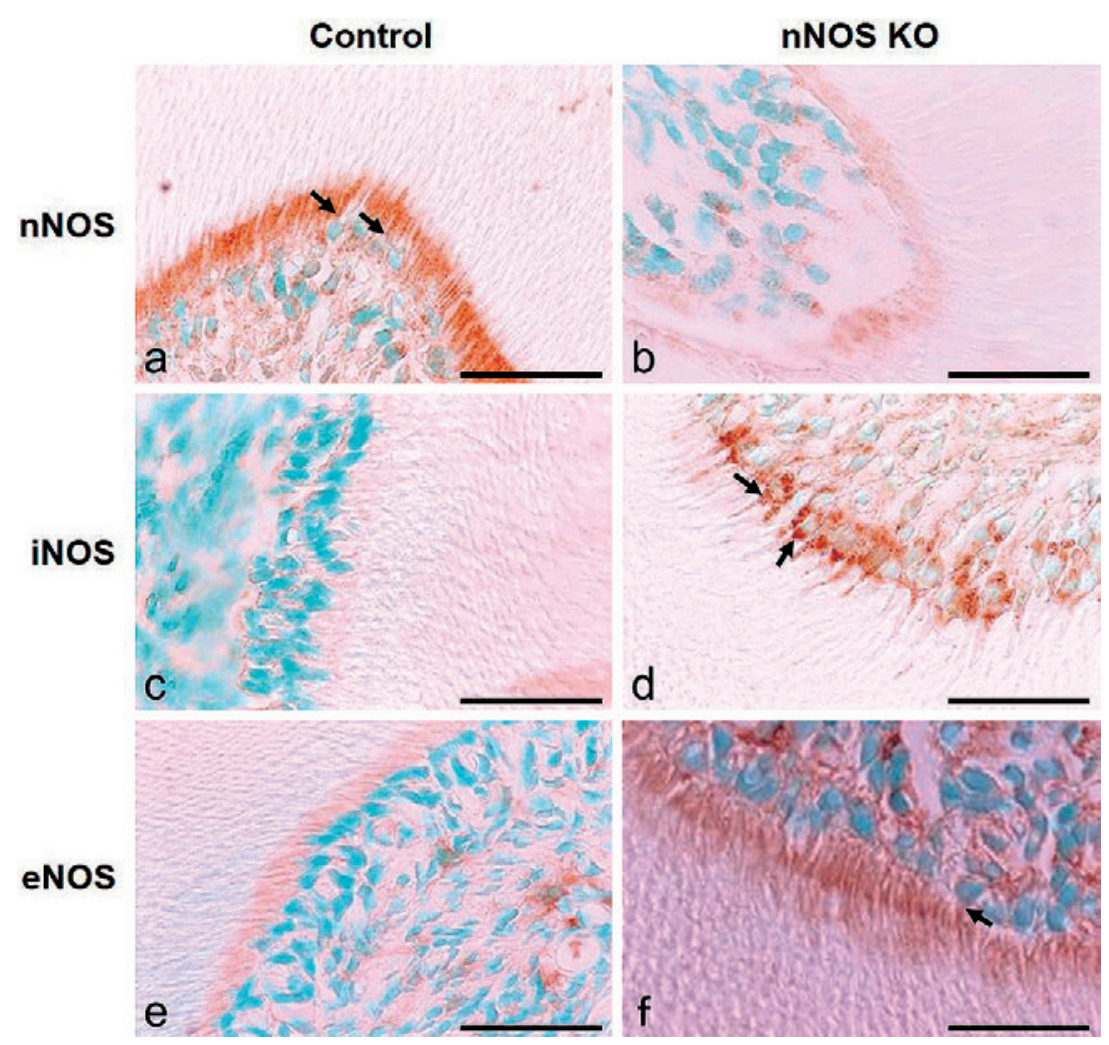

Fig. 2 NOS protein expression in odontoblasts

Positive reaction was observed for nNOS in control group (arrows) (a), whereas negative reaction was evident in $\mathrm{nNOS} \mathrm{KO}$ group $(\mathrm{b})$.

Negative expression of iNOS was observed in control group (c), whereas strongly positive expression was seen in basal region of cellular processes (arrows) in nNOS KO group (d). Weakly positive reaction was noted for eNOS in control group (e), and strongly positive reaction in basal region of cellular processes (arrow) in nNOS KO group.

Scale bar $=50 \mu \mathrm{m}$

Immunopositive staining is indicated by dark brown color.

control group (Fig. 1a). Staining was completely negative for nNOS protein in the nNOS KO group (Fig. 1b).

Negative expression of iNOS was observed in the central region of the dental pulp in the control group (Fig. 1c). In the nNOS KO group, a positive reaction revealing expression of iNOS was detected in endothelial cells and fibroblasts in the vicinity of blood vessels (Fig. 1d).

As for eNOS expression in the central region of the dental pulp, a weakly positive reaction was noted in endothelial cells and fibroblasts in the control group (Fig. 1e). In the nNOS KO group, the reaction was stronger in endothelial cells and fibroblasts (Fig. 1f) than in the control group.

A positive reaction revealing expression of nNOS was detected in odontoblasts in the control group (Fig. 2a). Staining was completely negative for nNOS protein in the nNOS KO group (Fig. 2b).

Negative iNOS expression was observed in odontoblasts in the control group (Fig. 2c). In the nNOS group, strongly positive iNOS staining was observed in the basal region of cellular processes in odontoblasts (Fig. 2d).

As for eNOS expression in odontoblasts, a 
weakly positive reaction was noted in the control group (Fig. 2e). In the nNOS KO group, the reaction was stronger in the basal region of cellular processes in odontoblasts (Fig. 2f) than in the control group.

\section{Discussion}

Expression of nNOS was positive in endothelial cells and fibroblasts in dental pulp in the control group. According to Law et al., NADPH-d was present in rat dental pulp, suggesting the presence of $\mathrm{nNOS}^{14)}$. Based on this earlier finding, immunohistochemical staining using anti-nNOS antibodies was performed in the present study to determine the presence or absence of nNOS in dental pulp. The results revealed a positive reaction for nNOS in endothelial cells and fibroblasts. This suggests that these cells are affected by nerve fibers containing nNOS, just as fibroblasts and endothelial cells are in other tissues.

Expression of eNOS and nNOS was positive in the basal region of odontoblasts in the control group. This was likely due to the special oxygen environment caused by protrusion of odontoblasts into dentinal tubules.

In the $\mathrm{nNOS} \mathrm{KO}$ mice, expression of $\mathrm{nNOS}$ was negative in endothelial cells and fibroblasts, which is not contradictory since nNOS was knocked out in these mice.

Negative iNOS expression was observed in endothelial cells and fibroblasts in dental pulp and odontoblasts in the control group. This is consistent with a previous immunohistochemical study which also reported negative expression of iNOS in normal dental pulp $^{4}$. In the nNOS KO mice in the present study, the reaction was positive or strongly positive in endothelial cells, fibroblasts, and odontoblasts. This suggests that iNOS compensated for nNOS deficiency against a decrease in NO production due to the absence of nNOS. The finding that iNOS produced much more NO than the other isoforms indicates that $\mathrm{NO}$ production increases with a decrease in nNOS. One earlier study found that iNOS increased in dental pulp during inflammation ${ }^{4)}$. However, the present results showed that iNOS increased when the balance of the various isoforms of NOS was broken by nNOS KO rather than inflammation.

Expression of eNOS was weakly positive in endothelial cells, fibroblasts, and odontoblasts in the control group. Previous immunohistochemical studies such as those by Felaco, M. et $a l^{5)}$ and Di Nardo et $a l^{4)}$ reported the presence of eNOS in human dental pulp, and similar results were obtained here, also. The reaction was more strongly positive in endothelial cells, fibroblasts, and odontoblasts in the nNOS KO mice than in the control mice. This suggests that the knocking out of nNOS increased expression of eNOS to maintain a constant NO level, indicating that eNOS compensates for nNOS deficiency.

Nitric oxide, reactive oxygen, and antioxidative enzymes form a complex network in the body. This study detected protein levels by immunohistological staining of cells, but investigation of mRNA by Northern blot and in situ hybridization may be necessary. The relationship between the expression of NOS and NO in various oral diseases remains unclear, and the production of, and pathology associated with NO have only been partially elucidated. The oral cavity is a space in which marked changes have been detected in NO levels, and each tissue is constantly influenced by NO. Nitric oxide is closely associated with the oral cavity, and further characterizing its role in this environment will require the application of a wide range of research methods in future study.

\section{Conclusion}

The results of this study confirmed that increased expression of iNOS and eNOS compensates for nNOS deficiency in vascular endothelial cells and fibroblasts in dental pulp and odontoblasts. 


\section{References}

1) Ambe K, Watanabe $H$, Takahashi $S$, Nakagawa T, Sasaki J (2016) Production and physiological role of NO in the oral cavity. Jpn Dent Sci Rev 52:14-21.

2) Brennan PA, Thomas GJ, Langdon JD (2003) The role of nitric oxide in oral diseases. Arch Oral Biol 48:93-100.

3) Brune B, von Knethen A, Sandau KB (1999) Nitric oxide (NO): an effector of apoptosis. Cell Death Differ 6:969-975.

4) Di Nardo Di Maio F, Lohinai Z, D'Arcangelo C, De Fazio PE, Speranza L, De Lutiis MA, Patruno A, Grilli A, Felaco M (2004) Nitric oxide synthase in healthy and inflamed human dental pulp. J Dent Res 83:312-316.

5) Felaco M, Di Maio FD, De Fazio P, D'Arcangelo C, De Lutiis MA, Varvara G, Grilli A, Barbacane RC, Reale M, Conti P (2000) Localization of the e-NOS enzyme in endothelial cells and odontoblasts of healthy human dental pulp. Life Sci 68:297-306.

6) Forstermann U, Boissel JP, Kleinert H (1998) Expressional control of the 'constitutive' isoforms of nitric oxide synthase (NOS I and NOS III). Faseb J 12:773-790.

7) Forstermann U, Mulsch A, Bohme E, Busse R (1986) Stimulation of soluble guanylate cyclase by an acetylcholine-induced endothelium-derived factor from rabbit and canine arteries. Circ Res 58:531-538.

8) Geller DA, Billiar TR (1998) Molecular biology of nitric oxide synthases. Cancer Metastasis Rev 17:7-23.

9) Ghalayani P, Jahanshahi G, Birang R, Bazazzadeh M (2013) Assessment of the effect of diode laser therapy on incisional wound healing and expression of iNOS and eNOS on rat oral tissue. Dent Res J (Isfahan) 10:348352.

10) Hecker M, Cattaruzza M, Wagner AH (1999) Regulation of inducible nitric oxide synthase gene expression in vascular smooth muscle cells. Gen Pharmacol 32:9-16.

11) Hesse M, Cheever AW, Jankovic D, Wynn TA (2000) NOS-2 mediates the protective antiinflammatory and antifibrotic effects of the Th1-inducing adjuvant, IL-12, in a Th2 model of granulomatous disease. Am J Pathol 157: 945-955.

12) Huang PL, Dawson TM, Bredt DS, Snyder SH, Fishman MC (1993) Targeted disruption of the neuronal nitric oxide synthase gene. Cell 75:1273-1286.
13) Ishioka M, Ishizuka $Y$, Shintani $S$, Yanagisawa T, Inoue T, Sasaki J, Watanabe H (2014) Expression profiles of NOS isoforms in gingiva of nNOS knockout mice. Tissue Cell 46:122126.

14) Law AS, Baumgardner KR, Meller ST, Gebhart GF (1999) Localization and changesin NADPHdiaphorase reactivity and nitric oxide synthase immunoreactivity in rat pulp following tooth preparation. J Dent Res 78:1585-1595.

15) MacMicking JD, Nathan C, Hom G, Chartrain N, Fletcher DS, Trumbauer M, Stevens K, Xie QW, Sokol K, Hutchinson N, et al. (1995) Altered responses to bacterial infection and endotoxic shock in mice lacking inducible nitric oxide synthase. Cell 81:641-650.

16) Nathan C, Xie QW (1994) Regulation of biosynthesis of nitric oxide. J Biol Chem 269: 13725-13728.

17) Shan J, Carbonara P, Karp N, Tulic M, Hamid Q Eidelman DH (2007) Localization and distribution of NOS1 in murine airways. Nitric Oxide 17:25-32.

18) Shimizu Y, Sakai M, Umemura Y, Ueda H (1997) Immunohistochemical localization of nitric oxide synthase in normal human skin: expression of endothelial-type and inducibletype nitric oxide synthase in keratinocytes. J Dermatol 24:80-87.

19) Son H, Hawkins RD, Martin K, Kiebler M, Huang PL, Fishman MC, Kandel ER (1996) Long-term potentiation is reduced in mice that are doubly mutant in endothelial and neuronal nitric oxide synthase. Cell 87:10151023.

20) Takaki A, Morikawa K, Tsutsui M, Murayama Y, Tekes E, Yamagishi H, Ohashi J, Yada T, Yanagihara N, Shimokawa H (2008) Crucial role of nitric oxide synthases system in endothelium-dependent hyperpolarization in mice. J Exp Med 205:2053-2063.

21) Wei XQ, Charles IG, Smith A, Ure J, Feng GJ, Huang FP, Xu D, Muller W, Moncada S, Liew FY (1995) Altered immune responses in mice lacking inducible nitric oxide synthase. Nature 375:408-411.

\section{Correspondence:}

Dr. Yoichi Ishizuka

Department of Epidemiology and Public Health, Tokyo Dental College 2-9-18 Kanda-Misakicho, Chiyoda-ku, Tokyo 101-0061, Japan

E-mail: ishizukayouichi@tdc.ac.jp 